\title{
Energetic Balance for the Flow Induced by a Constantly Accelerating Plate in a Second Grade Fluid
}

\author{
Corina Fetecau ${ }^{1}$, Masood Khan ${ }^{2}$, Constantin Fetecau ${ }^{3}$ \\ ${ }^{1}$ Department of Theoretical Mechanics, Technical University, Iasi, Romania \\ ${ }^{2}$ Department of Mathematics, Quaid-i-Azam University, Islamabad, Pakistan \\ ${ }^{3}$ Department of Mathematics, Technical University, Iasi, Romania \\ E-mail: cfetecau@yahoo.de,\{mkhan_21,fetecau_constantin\}@yahoo.com \\ Received February 4, 2010; revised March 21, 2010; accepted March 25, 2010
}

\begin{abstract}
Dissipation, power due to the shear stress at the wall and the boundary layer thickness corresponding to the unsteady flow of a second grade fluid, due to a constantly accelerating plate, are established in exact and approximate forms. The changing of the kinetic energy with time is also determined from the energetic balance. Exact expressions of the same entities for Newtonian fluids are recovered as limiting cases of general results.
\end{abstract}

Keywords: Second Grade Fluid, Dissipation, Energetic Balance, Power, Kinetic Energy, Constantly Accelerating Plate

\section{Introduction}

In recent years, the interest for flows of non-Newtonian fluids has considerably increased, while the energetic aspects regarding these motions are scarcely met in the literature. To the best of our knowledge, the first results of this kind seem to be those of Bühler and Zierep [1] concerning the Rayleigh-Stokes problem for Newtonian fluids. These results have been recently extended to second grade and Maxwell fluids by Zierep and Fetecau $[2,3]$. In these works, the authors also determine the boundary layer thickness and use it in order to obtain series solutions for the velocity field and the shear stress.

The aim of this note is to provide the energetic study for the unsteady flow of a second grade fluid, driven by the transversal motion of a constantly accelerating plate, and to compare the obtained results with those corresponding to the flow induced by a suddenly moved plate (Stokes' first problem). Of special interest is the energetic balance of the three terms: Changing of the kinetic energy with time, Dissipation and the Power due to the shear stress at the wall. The last term describes the input energy that is necessary to keep the medium running. A decisive question is whether these terms are larger or smaller than in the Newtonian case.

\section{Statement of the Problem}

The flows to be here considered have the velocity field

$$
\mathbf{v}=\mathbf{v}(y, t)=u(y, t) \mathbf{i}
$$

where $\mathbf{i}$ is the unit vector along the $\mathrm{x}$-direction of the Cartesian coordinate system $\mathrm{x}, \mathrm{y}$ and $\mathrm{z}$. For such motions, the constraint of incompressibility is automatically satisfied while the governing equations, in the absence of body forces and a pressure gradient in the flow direction, are $[2,4]$

$$
\begin{aligned}
\partial_{t} u(y, t) & =\left(v+\alpha \partial_{t}\right) \partial_{y}^{2} u(y, t), \\
\tau(y, t) & =\left(\mu+\alpha_{1} \partial_{t}\right) \partial_{y} u(y, t),
\end{aligned}
$$

where $\mu$ is the dynamic viscosity, $\alpha_{1}$ is a material constant, $v=\mu / \rho$ is the kinematic viscosity of the fluid ( $\rho$ being its constant density), $\alpha=\alpha_{1} / \rho$ and $\tau=S_{x y}$ is the non-trivial shear stress.

The appropriate initial and boundary conditions, corresponding to the motion over an infinite constantly accelerating plate, are given by [4-6]

$$
\begin{aligned}
& u(y, 0)=0, y>0 ; u(0, t)= \\
& A t, t \geq 0 ; u(y, t), \partial_{y} u(y, t) \rightarrow 0 \text { as } y \rightarrow \infty,
\end{aligned}
$$

where $A$ is a constant.

The energetic balance for an infinite volume of rectangular cross-section with $x \in[0, \ell]$ and $z \in[0,1]$, as it results from [1-3], is given by the identity

$$
\frac{d}{d t} E_{k i n}+L+\Phi=0
$$


where $E_{\text {kin }}$ is the kinetic energy and

$$
\begin{aligned}
& L=-\ell u(0, t) \tau(0, t)= \\
& -\ell u_{w}(t) \tau_{w}(t), \Phi=\int_{0}^{\infty} \tau(y, t) \frac{\partial u(y, t)}{\partial y} d y,
\end{aligned}
$$

are the power of the shear stress at the wall and the dissipation.

The boundary layer thickness $\delta$, as it results from [7], is given by

$$
\delta=\frac{1}{u(0, t)} \int_{0}^{\infty} u(y, t) d y
$$

and represents the thickness of the fluid layer moved with the plate by friction. One measure of the boundary layer thickness is the distance from the wall where the velocity of the fluid differs by $1 \%$ from the external velocity.

\section{Exact Results}

Let us consider an incompressible second grade fluid at rest over an infinitely extended flat plate situated in the $(x, z)$ - plane. After time $t=0$ the plate is subjected to a transversal motion, in its plane, of velocity $u(0, t)=A t$ in the $x$-direction. Owing to the shear, the fluid over the plate is gradually moved, its velocity being of the form (1). The governing equation is given by Equation (2) while the corresponding initial and boundary conditions are of the form (3).

The velocity field $u(y, t)$ and the adequate shear stress $\tau(y, t)$ corresponding to this problem, as it results from [8] (see Equations (3) and (4)), are given by (see also [5], Equation (5) with $V(t)=A t$ or [6], Equation (7) for the velocity field only)

$$
\begin{gathered}
u(y, t)= \\
A t-\frac{2 A}{v \pi} \int_{0}^{\infty}\left[1-\exp \left(-\frac{v t \xi^{2}}{1+\alpha \xi^{2}}\right)\right] \frac{\sin (y \xi)}{\xi^{3}} d \xi, \\
\tau(y, t)= \\
-\frac{2 \rho A}{\pi} \int_{0}^{\infty}\left[1-\frac{1}{1+\alpha \xi^{2}} \exp \left(-\frac{v t \xi^{2}}{1+\alpha \xi^{2}}\right)\right] \frac{\cos (y \xi)}{\xi^{2}} d \xi .
\end{gathered}
$$

Introducing (7) and (8) into (5) and (6), we find for $L$, $\Phi$ and $\delta$ the next exact expressions

$$
\begin{aligned}
& L= \\
& -\frac{2 \rho \ell A^{2} t}{\pi} \int_{0}^{\infty}\left[1-\frac{1}{1+\alpha \xi^{2}} \exp \left(-\frac{v t \xi^{2}}{1+\alpha \xi^{2}}\right)\right] \frac{1}{\xi^{2}} d \xi,
\end{aligned}
$$

$$
\begin{aligned}
\Phi & =\frac{4 \rho \ell A^{2}}{v \pi^{2}} \int_{0}^{\infty}\left\{\int_{0}^{\infty}\left[1-\exp \left(-\frac{v t \xi^{2}}{1+\alpha \xi^{2}}\right)\right] \frac{\cos (y \xi)}{\xi^{2}} d \xi\right. \\
& \left.\times \int_{0}^{\infty}\left[1-\frac{1}{1+\alpha \xi^{2}} \exp \left(-\frac{v t \xi^{2}}{1+\alpha \xi^{2}}\right)\right] \frac{\cos (y \xi)}{\xi^{2}} d \xi\right\} d y,
\end{aligned}
$$

$\delta=$

$$
\int_{0}^{\infty}\left\{1-\frac{2}{v \pi t} \int_{0}^{\infty}\left[1-\exp \left(-\frac{v t \xi^{2}}{1+\alpha \xi^{2}}\right)\right] \frac{\sin (y \xi)}{\xi^{3}} d \xi\right\} d y .
$$

By making $\alpha_{1}$ and thereby $\alpha \rightarrow 0$ into Equations (7)-(11), we attain to the similar expressions

$$
\begin{gathered}
u_{N}(y, t)= \\
A t-\frac{2 A}{v \pi} \int_{0}^{\infty}\left(1-\mathrm{e}^{-v t \xi^{2}}\right) \frac{\sin (y \xi)}{\xi^{3}} d \xi, \\
\tau_{N}(y, t)= \\
-\frac{2 \rho A}{\pi} \int_{0}^{\infty}\left(1-\mathrm{e}^{-v t \xi^{2}}\right) \frac{\cos (y \xi)}{\xi^{2}} d \xi, \\
L_{N}=-\frac{2 \rho \ell A^{2} t}{\pi} \int_{0}^{\infty}\left(1-\mathrm{e}^{-v t \xi^{2}}\right) \frac{1}{\xi^{2}} d \xi, \\
\Phi_{N}=\int_{0}\left[\int_{0}^{\infty}\left(1-\mathrm{e}^{-v t \xi^{2}}\right) \frac{\cos (y \xi)}{\xi^{2}} d \xi\right]^{2} d y, \\
\delta_{N}= \\
\int_{0}^{\infty}\left[1-\frac{2}{v \pi t} \int_{0}^{\infty}\left(1-\mathrm{e}^{-v t \xi^{2}}\right) \frac{\sin (y \xi)}{\xi^{3}} d \xi\right] d y,
\end{gathered}
$$

corresponding to a Newtonian fluid performing the same motion. The integrals in (12)-(14) can be evaluated to give the classical solutions

$$
\begin{aligned}
& u_{N}(y, t)=4 A t \mathrm{i}^{2} \operatorname{Erfc}\left(\frac{y}{2 \sqrt{v t}}\right), \tau_{N}(y, t)= \\
& -2 \rho A \sqrt{v t} \mathrm{i} \operatorname{Erfc}\left(\frac{y}{2 \sqrt{v t}}\right),
\end{aligned}
$$

for velocity and shear stress and the simple expressions [9, Equations (13) and (14)]

$$
\begin{gathered}
L_{N}=-2 \rho \ell A^{2} t \sqrt{\frac{v t}{\pi}}, \Phi_{N}=\frac{8(\sqrt{2}-1)}{3} \rho \ell A^{2} t \sqrt{\frac{v t}{\pi}}, \delta_{N}=\frac{4}{3} \sqrt{\frac{v t}{\pi}}, \\
\frac{d}{d t} E_{k i n}^{N}=\frac{2(7-4 \sqrt{2})}{3} \rho \ell A^{2} t \sqrt{\frac{v t}{\pi}}
\end{gathered}
$$

for $L_{N}, \Phi_{N}, \delta_{N}$ and $d E_{k i n}^{N} / d t$. Into Equation (15) 


$$
\begin{aligned}
& \mathrm{i}^{n} \operatorname{Erfc}(x)= \\
& \int_{x}^{\infty} \mathrm{i}^{n-1} \operatorname{Erfc}(\xi) d \xi \text { with } n= \\
& 0,1,2, \ldots ; \mathrm{i}^{0} \operatorname{Erfc}(x)=\operatorname{Erfc}(x),
\end{aligned}
$$

are the integrals of the complementary error function $\operatorname{Erfc}(\cdot)$.

\section{Asymptotic approximations for $(\alpha / v t)<<1$}

Let us now consider the case when the material module $\alpha_{1}$ is small enough, so that the dimensionless memory variable $\alpha /(v t)$ is much less than one. Simple computations show that the next approximations

$$
\begin{gathered}
\exp \left(-\frac{v t \xi^{2}}{1+\alpha \xi^{2}}\right)=\mathrm{e}^{-v t \xi^{2}} \exp \left(\frac{\alpha v t \xi^{4}}{1+\alpha \xi^{2}}\right)= \\
\mathrm{e}^{-v t \xi^{2}}\left[1+\alpha v t \xi^{4}+\mathrm{O}\left(\alpha^{2}\right)\right] \\
\frac{1}{1+\alpha \xi^{2}} \exp \left(-\frac{v t \xi^{2}}{1+\alpha \xi^{2}}\right)= \\
\mathrm{e}^{-v t \xi^{2}}\left[1+\alpha \xi^{2}\left(v t \xi^{2}-1\right)+\mathrm{O}\left(\alpha^{2}\right)\right]
\end{gathered}
$$

are valid for each $\xi$ and $t$ greater zero.

Introducing Equations (17) and (18) into (9)-(11) and having in mind Equation (16), we can easy prove that

$$
L=L_{N}+\alpha \frac{2 \rho \ell A^{2} t}{\pi} \int_{0}^{\infty}\left(v t \xi^{2}-1\right) \mathrm{e}^{-v t \xi^{2}} d \xi+\mathrm{O}\left(\alpha^{2}\right),
$$

$$
\Phi=\Phi_{N}+\alpha \frac{4 \rho \ell A^{2}}{v \pi^{2}} \int_{0}^{\infty}\left[\int_{0}^{\infty} \frac{1-\mathrm{e}^{-v t \xi^{2}}}{\xi^{2}} \cos (y \xi) d \xi \int_{0}^{\infty}\left(2 v t \xi^{2}-1\right) \mathrm{e}^{-v t \xi^{2}} \cos (y \xi) d \xi\right] d y+\mathrm{O}\left(\alpha^{2}\right)
$$

$$
\delta=\delta_{N}+\alpha \frac{2}{\pi} \int_{0}^{\infty} \int_{0}^{\infty} \xi \mathrm{e}^{-v t \xi^{2}} \sin (y \xi) d \xi d y+\mathrm{O}\left(\alpha^{2}\right) .
$$

Evaluating all integrals, as well as in [2] and [3], we attain to the following approximate expressions

$$
\begin{gathered}
L=L_{N}\left\{1+\frac{1}{4} \frac{\alpha}{v t}+\mathrm{O}\left[\left(\frac{\alpha}{v t}\right)^{2}\right]\right\}, \\
\delta=\delta_{N}\left\{1+\frac{3}{4} \frac{\alpha}{v t}+\mathrm{O}\left[\left(\frac{\alpha}{v t}\right)^{2}\right]\right\} . \\
\Phi=\Phi_{N}\left\{1+\frac{3(2-\sqrt{2})}{8} \frac{\alpha}{v t}+\mathrm{O}\left[\left(\frac{\alpha}{v t}\right)^{2}\right]\right\},
\end{gathered}
$$

Equations (4), (16), (22) and (23), also imply

$$
\frac{d}{d t} E_{k i n}=\frac{d}{d t} E_{k i n}^{N}\left\{1+\frac{9(5-2 \sqrt{2})}{68} \frac{\alpha}{v t}+\mathrm{O}\left[\left(\frac{\alpha}{v t}\right)^{2}\right]\right\} \text {. }
$$

The results corresponding to $L, \Phi$ and $d E_{k i n} / d t$ are in accordance with those obtained in [2] for the motion due to a suddenly moved plate. More exactly, into both motions, $L, \Phi$ and $d E_{k i n} / d t$ increase for second grade fluids in comparison with Newtonian fluids. On the other hand the boundary layer thickness $\delta$ increases for the present motion and decreases for the first problem of Stokes.

\section{Analogy to the Teipel Series Expansion}

As early as 1981, Teipel [7] studied the first problem of Stokes for second grade fluids and provided a series so- lution. This series has been determined until an arbitrary constant, although all initial and boundary conditions have been fulfilled. In order to determine that constant, Teipel used a new initial condition, namely $\delta(0)=0$. Later, in order to determine this constant, Erdogan and Imrak [10] used the condition that the volume flux is zero at time $t=0$.

Following the Teipel's idea [7], and having in mind Equation (3), we are looking for a velocity field of the form

$$
\begin{aligned}
& u(y, t)= \\
& A t\left\{f_{0}(\eta)+\frac{\alpha}{v t} f_{1}(\eta)+\mathrm{O}\left[\left(\frac{\alpha}{v t}\right)^{2}\right]\right\} ; \eta=\frac{y}{2 \sqrt{v t}},
\end{aligned}
$$

for the flow induced by a constantly accelerating plate in a second grade fluid. Introducing (25) into the governing Equation (2) and identifying the coefficients of the same powers, we find that

$$
\begin{aligned}
& f_{0}^{\prime \prime}(\eta)+2 \eta f_{0}^{\prime}(\eta)-4 f_{0}(\eta)= \\
& 0 \text { and } f_{1}^{\prime \prime}(\eta)+2 \eta f_{1}^{\prime}(\eta)=\frac{\eta}{2} f_{0}^{\prime \prime \prime}(\eta)
\end{aligned}
$$

The appropriate boundary conditions being

$$
f_{0}(0)=1, f_{0}(\infty)=0 \text { and } f_{1}(0)=f_{1}(\infty)=0,
$$

it results that, in contrast with the results of Teipel, the functions $f_{0}(\cdot)$ and $f_{1}(\cdot)$ are completely determined and have the forms

$$
f_{0}(\eta)=4 \mathrm{i}^{2} \operatorname{Erfc}(\eta), \quad f_{1}(\eta)=\frac{\eta}{\sqrt{\pi}} e^{-\eta^{2}}
$$

Consequently, an approximate solution for the velocity field is 
$u(y, t)=$

$4 A t \mathrm{i}^{2} \operatorname{Erfc}\left(\frac{y}{2 \sqrt{v t}}\right)+\frac{\alpha}{v t} \frac{A y}{2} \sqrt{\frac{t}{v \pi}} \exp \left(-\frac{y^{2}}{4 v t}\right)+\mathrm{O}\left[\left(\frac{\alpha}{v t}\right)^{2}\right]$.

Introducing (29) into (2) and keeping the terms of the first order into $\alpha /(v t)$, we get for the shear stress the approximate expression

$$
\begin{aligned}
& \tau(y, t)= \\
& -2 \rho A \sqrt{v t} \mathrm{i} \operatorname{Erfc}\left(\frac{y}{2 \sqrt{v t}}\right)- \\
& \frac{\alpha}{v t} \frac{\rho A}{2} \sqrt{\frac{v t}{\pi}}\left(1+\frac{y^{2}}{2 v t}\right) \exp \left(-\frac{y^{2}}{4 v t}\right)+\mathrm{O}\left[\left(\frac{\alpha}{v t}\right)^{2}\right] .
\end{aligned}
$$

\section{Conclusions}

In this note, the power due to the shear stress at the wall, dissipation and the boundary layer thickness corresponding to the unsteady flow of a second grade fluid induced by a constantly accelerating plate, have been determined in exact and approximate forms. Their exact expressions corresponding to Newtonian fluids, performing the same motion, are recovered as limiting cases of general results. As a consequence, the changing of the kinetic energy with time is obtained from the energetic balance. Finally, series solutions are established both for the velocity field $u(y, t)$ and the shear stress $\tau(y, t)$.

In conclusion, from Equations (22)-(24) it clearly results that $L, \Phi, \delta$ and $d E_{k i n} / d t$ increase for second grade fluids in comparison with Newtonian fluids. Furthermore, the results corresponding to $L, \Phi$ and $d E_{\text {kin }} / d t$ are in accordance with those obtained in [2] for the motion due to a suddenly moved plate. As regards the boundary layer thickness, the present result is opposite to that from [2], where $\delta$ decreases for second grade fluids in comparison with the Newtonian fluids.

Finally, in order to reveal some relevant physical aspects of the obtained results, the diagrams of the velocity $u(y, t)$ and the shear stress $\tau(y, t)$ against $y$ are presented for different values of the time $t$. Figures 1 and 2 clearly show that the approximate solutions (29) and (30) give a very good approximation for the exact solutions (7) and (8). The variations of the power due to the shear stress at the wall $L(t)$ and the boundary layer thickness $\delta(t)$ are also depicted in Figures $\mathbf{3}$ and $\mathbf{4}$ for different values of the material constant $\alpha$. These graphs show that $L(t)$ and $\delta(t)$ are increasing functions with respect to $t$ and decreasing ones with regard to $\alpha$. Furthermore, as expected, for $\alpha \rightarrow 0$ they are going to $L_{N}$ and $\delta_{N}$ corresponding to Newtonian fluids.

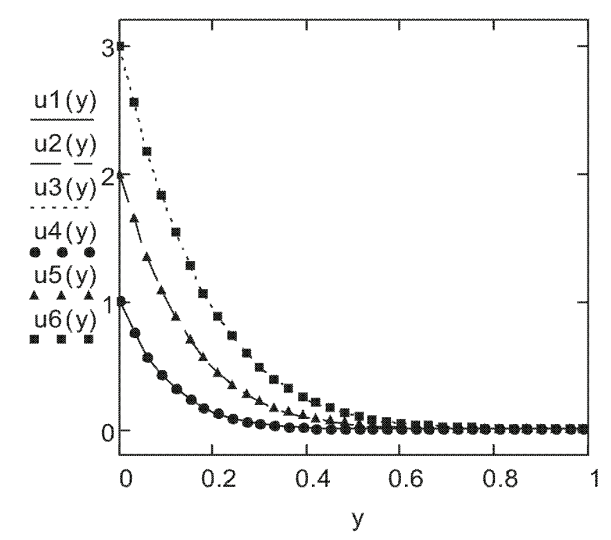

Figure 1. Comparison between the exact solution (7)curves $u 1(y), u 2(y), u 3(y)$ and the asymptotic solution (29)curves u3(y), u4(y), u5(y), for $A=0.2, v=0.003, \rho=972$, $\alpha=\mathbf{0 . 0 0 2}$ and different values of $t$.

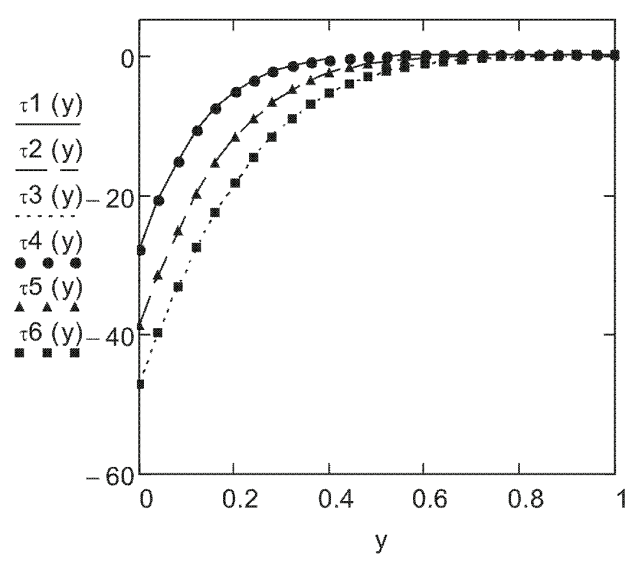

Figure 2. Comparison between the exact solution (8)curves $\tau \mathbf{1}(y), \tau \mathbf{2 ( y )}, \tau \mathbf{4}(y)$ and the asymptotic solution (29)—curves $\tau 3(y), \tau 4(y), \tau 5(y)$, for $A=0.2, v=0.003$, $\rho=972, \alpha=0.002$ and different values of $t$.

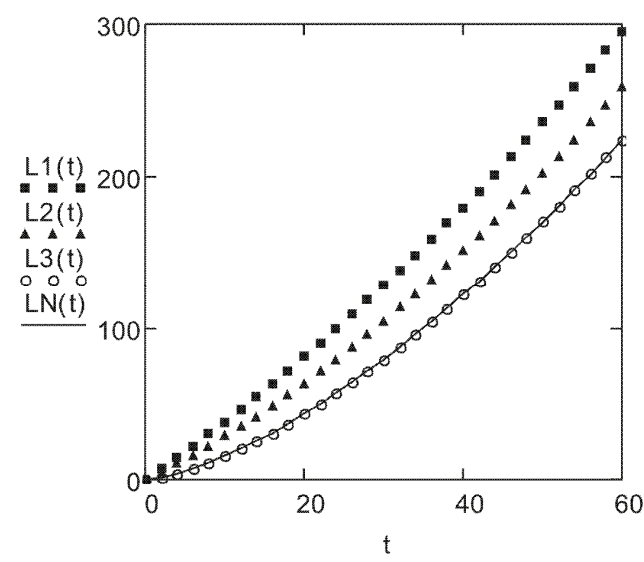

Figure 3. Power of the shear at the wall $L(t)$, corresponding to Newtonian fluid-curve $L N(t)$ and second grade fluidcurves $L 1(t), L 2(t), L 3(t)$, for $A=0.2, \quad v=0.003, \quad \rho=972$, and different values of $\alpha$. 


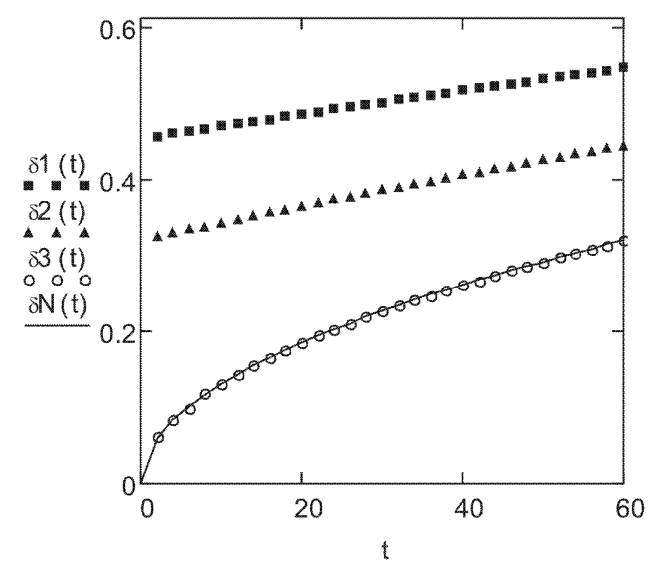

Figure 4. The boundary layer thickness $\delta(t)$, corresponding to Newtonian fluid-curve $\delta N(t)$ and second grade fluid-curves $\delta \mathbf{1}(t), \delta \mathbf{2}(t), \delta 3(t)$, for $v=\mathbf{0 . 0 0 3}$ and different values of $\alpha$.

\section{Acknowledgements}

The authors Corina Fetecau and Constantin Fetecau acknowledge support from the Ministry of Education and Research, CNCSIS, through PN II-Ideas, Grant No. 26/2809-2007, Cod CNCSIS ID_593.

The authors also would like to express their gratitude to the referees both for their careful assessment and the fruitful comments and suggestions regarding the first form of this note.

\section{References}

[1] K. Bühler and J. Zierep, "Energetische Betrachtungen Zum Rayleigh-Stokes Problem," Proceedings in Applied Mathematics and Mechanics, Vol. 5, No. 1, 2005, pp. 539-540.
[2] J. Zierep and C. Fetecau, "Energetic Balance for the Rayleigh-Stokes Problem of a Second Grade Fluid," International Journal of Engineering Science, Vol. 45, No. 1, 2007, pp. 155-162.

[3] J. Zierep and C. Fetecau, "Energetic Balance for the Rayleigh-Stokes Problem of a Maxwell Fluid," International Journal of Engineering Science, Vol. 45, No. 2-8, 2007, pp. 617-627.

[4] R. Bandelli, K. R. Rajagopal and G. P. Galdi, "On some Unsteady Motions of Fluids of Second Grade," Archives of Mechanics, Vol. 47, No. 4, 1995, pp. 661-676.

[5] C. Fetecau and J. Zierep, "On a Class of Exact Solutions of the Equations of Motion of a Second Grade Fluid," Acta Mechanica, Vol. 150, No. 1-2, 2001, pp. 135-138.

[6] M. E. Erdogan and C. E. Imrak, "On Unsteady Motions of a Second-Order Fluid over a Plane Wall," International Journal of Non-Linear Mechanics, Vol. 38, No. 7, 2003, pp. 1045-1051.

[7] I. Teipel, "The Impulsive Motion of a Flat Plate in a Visco-Elastic Fluid," Acta Mechanica, Vol. 39, No. 3-4, 1981, pp. 277-279.

[8] C. Fetecau, S. C. Prasad and K. R. Rajagopal, “A Note on the Flow Induced by a Constantly Accelerated Plate in an Oldroyd-B Fluid," Applied Mathematical Modelling, Vol. 31, No. 4, 2007, pp. 647-654.

[9] C. Fetecau, D. Vieru, A. Mahmood and C. Fetecau, "On the Energetic Balance for the Flow of a Maxwell Fluid Due to a Constantly Accelerating Plate," Acta Mechanica, Vol. 203, No. 1-2, 2009, pp. 89-96.

[10] M. E. Erdogan and C. E. Imrak, "On the Comparison of Two Different Solutions in the Form of Series of the Governing Equation of an Unsteady Flow of a Second Grade Fluid," International Journal of Non-Linear Mechanics, Vol. 40, No. 4, 2005, pp. 545-550. 